\title{
Bilateral Impacted Maxillary Canine with Left Lateral Incisor Root Resorption
}

\section{K S Negi*}

Department of Orthodontics and Dentofacial Orthopedics, HP Government Dental College and Hospital, India

\begin{abstract}
After the third molars, the maxillary permanent canines are the teeth most frequently impacted. The diagnosis, based on clinical and radiographic examination, is usually made by the general dental practitioner. An ectopic canine, if undiagnosed and untreated, will induce complex complication like root resorption of adjacent tooth or teeth. Periodic examination starting at age 8 , including clinical intraoral palpation and selective radiographs, may aid in the early diagnosis of unerupting and potentially impacted permanent canines. When such a diagnosis is apparent, timely interceptive therapy may then be instituted to avoid a situation like impaction and resortion of root of adjacent tooth. This paper presents brief review and orthodontic management of bilaterally; both palatal as well as labilally impacted canines with advanced resorption of adjacent lateral incisor on left side.
\end{abstract}

Keywords: Impacted canine; Root resorption; Closed flap technique Introduction

Tooth impaction can be defined as the infraosseous position of the tooth after the expected time of eruption, whereas the anomalous infraosseous position of the canine before the expected time of eruption can be defined as a displacement. Most of the time, palatal displacement of the maxillary canine results in impaction [1]. Disturbances in the eruption of permanent maxillary canines are common because they develop deep within the maxilla and have the longest path to travel compared with any other tooth in the oral cavity. Canines play a vital role in facial appearance, dental esthetics, arch development and functional occlusion. As a result, orthodontists have acknowledged the significance of retaining impacted maxillary canines and have proposed various techniques to effectively and efficiently recover these teeth. In orthodontics and dentistry in general, canine impaction is a dental anomaly that occurs frequently, and clinicians must be prepared to manage it [2].

\section{Prevalence and etiology}

Maxillary canines are the most commonly impacted teeth, second only to third molars [3,4]. Maxillary canine impaction occurs in approximately 2 percent of the population and is twice as common in females as it is in males $[5,6]$. The incidence of canine impaction in the maxilla is more than twice that in the mandible [7]. Of all patients who have impacted maxillary canines, 8 percent have bilateral impactions [4]. Approximately one third of impacted maxillary canines are located labially, and two-thirds are located palatally [8,9]. Canine impaction can be caused by various factors. The exact etiology of palatally displaced maxillary canines is unknown. The results of Jacoby's [10] study showed that 85 percent of palatally impacted canines had sufficient space for eruption, whereas only 17 percent of labially impacted canines had sufficient space. Therefore, arch length discrepancy is thought to be a primary etiologic factor for labially impacted canines [9]. Several etiologic factors for canine impactions have been proposed [3-13]:

Localized: Tooth size-arch length discrepancies, Failure of the primary canine root to resorb, Prolonged retention or early loss of the primary canine, Ankylosis of the permanent canine, Cyst or neoplasm, Dilaceration of the root, Absence of the maxillary lateral incisor, Variation in root size of the lateral incisor (that is, peg-shaped lateral incisor), Variation in timing of lateral incisor root formation, Iatrogenic factors, Idiopathic factors.
Systemic: Endocrine deficiencies, Febrile diseases, Irradiation.

Genetic: Heredity, Malposed tooth germ, Presence of an alveolar cleft.

Two major theories associated with palatally displaced maxillary canines are the guidance theory and genetic theory. The guidance theory proposes that the canine erupts along the root of the lateral incisor, which serves as a guide, and if the root of the lateral incisor is absent or malformed, the canine will not erupt [11]. The genetic theory points to genetic factors as a primary origin of palatally displaced maxillary canines and includes other possibly associated dental anomalies, such as missing or small lateral incisors [12]. Baccetti [13] reported that palatally impacted maxillary canines are genetically reciprocally associated with anomalies such as enamel hypoplasia, infraocclusion of primary molars, aplasia of second premolars and small maxillary lateral incisors. Peck et al. [12] stressed that the high probability of additional dental abnormalities' occurring in combination with a palatally displaced canine-such as congenital tooth absence and delayed eruption-should alert clinicians to be circumspect when planning treatment. Becker [11] reported an increase of 2.4 times in the incidence of palatally impacted canines adjacent to the sites of missing lateral incisors compared with palatally impacted canines in the general population. It remains uncertain, however, whether an anomalous lateral incisor is a local causal factor for palatally displaced canines or the displaced canines are the result of an associated genetic developmental influence.

\section{Sequelae of maxillary canine impaction}

Impacted canines usually are asymptomatic. Therefore, a patient usually is unaware of the impacted canines existence. General practitioners and orthodontists discover most of these impacted

${ }^{*}$ Corresponding author: K S Negi, Department of Orthodontics and Dentofacia Orthopedics, HP Government Dental College and Hospital, India, E-mail docksnortho@yahoo.com

Received November 10, 2011; Accepted April 24, 2012; Published April 28, 2012

Citation: Negi KS (2012) Bilateral Impacted Maxillary Canine with Left Lateral Incisor Root Resorption. Dentistry 2:134. doi:10.4172/2161-1122.1000134

Copyright: ( 2012 Negi KS. This is an open-access article distributed under the terms of the Creative Commons Attribution License, which permits unrestricted use, distribution, and reproduction in any medium, provided the original author and source are credited. 
teeth during initial radiographic examinations. Sequelae of abnormal eruption paths within the dentoalveolar process can include impactions and have serious clinical ramifications. For example, labially or palatally impacted teeth cause migration of the neighboring teeth and loss of arch length. In addition, unerupted canines may increase the patient's risk of developing a cystic lesion and infection and cause root resorption of the nearby lateral incisors and jeopardize the longevity of lateral incisors [14]. Lateral incisors adjacent to ectopically erupted canines have an incisor root resorption incidence of approximately 0.7 percent, but even with continued root development, an abnormally erupting canine can harm the adjacent lateral incisor $[14,15]$. On the other hand, the presence of the impacted canine may cause no untoward effects during the patient's lifetime. The potential complications, however, emphasize the need for dentists to monitor the development and eruption of impacted canines closely during routine dental examinations of growing children.

\section{Clinical diagnosis}

Various clinical signs of canine impaction are documented in the dental literature. These signs include delayed eruption of the permanent canine, overretention of the primary canine, absence of a labial bulge, presence of a palatal bulge and distal crown tipping of the lateral incisor [4]. Kuro and Ericson [14] suggested that absence of the "canine bulge" when the child is around 11 years of age is not an indication of canine impaction. However, they suggested palpation of the buccal surface of the alveolar process distal to the lateral incisor to help determine the position of the maxillary canine before its emergence [11]. If a labial bulge is absent in a 9- or 10-year-old patient, eruption disturbance of the permanent canine should be suspected and a radiograph obtained to confirm the diagnosis $[4,11]$.

\section{Radiographic diagnosis}

Several methods have been used to radiographically evaluate impacted maxillary canines. These methods include intraoral techniques (occlusal and periapical projections) and extraoral techniques (panoramic, posteroanterior or lateral cephalometric radiographs). The most practical method of obtaining an occlusal radiograph is by positioning the $\mathrm{x}$-ray tube directly over the bridge of the nose, at a 60-degree angle to the occlusal plane [11]. This method has been used to determine the buccolingual position of impacted teeth. However, the traditional method of locating impacted teethspecifically, maxillary canines-has been the use of a two-dimensional technique with periapical radiographs, known as the buccal object rule [4]. This technique consists of taking two periapical radiographs at different mesiodistal angulations and using the same-lingualopposite buccal (SLOB) rule to determine the tooth's buccolingual position. The radiographic interpretation of the SLOB rule is if, when obtaining the second radiograph, the clinician moves the $\mathrm{x}$-ray tube in a distal direction, and on the radiograph the tooth in question also moves distally, then the tooth is located on the lingual or palatal side. Accordingly, if the impacted canine is located buccally, the crown of the tooth moves mesially. When children are 8 or 9 years of age, dentists can locate the children's maxillary canines easily on lateral cephalometric radiographs. The inclination of the maxillary canines should be parallel to that of the maxillary incisors. In posteroanterior radiographs, the canines should be angled medially, and the crowns should be located below the apexes of the lateral incisors and well below the lateral border of the nasal cavity. The canine roots should be located laterally to the lateral border of the nasal cavity. If a canine is angled medially, with the crown located medially to the lateral border of the nasal cavity, the possibility of impaction should be considered [11]. Assessing the position of the impacted canine is key to determining the feasibility of and proper access for a surgical procedure, as well as the best direction for application of orthodontic forces [4]. The crown of the ectopically erupting canine may put pressure on the lateral incisor root, causing it to resorb. Clinicians can localize canines by using advanced three-dimensional imaging techniques. Cone-beam computed tomography (CBCT) can identify and locate the position of impacted canines accurately. By using this imaging technique, dentists also can assess any damage to the roots of adjacent teeth and the amount of bone surrounding each tooth. In a study, Liu et al. [16] used $\mathrm{CBCT}$ to evaluate variations in location of impacted maxillary canines. They found that the position of impacted maxillary canines varies greatly. Reports of maxillary canine impactions vary considerably in orientation, and CBCT provides information to dentists so that they can properly manage impacted canines surgically and orthodontically $[17,18]$. However, increased cost, time, radiation exposure and medicolegal issues associated with using CBCT limit its routine use [19].

\section{Case Report}

A 14 year old female referred by a general dentist to the orthodontic clinic of H.P. Govt. Dental College Shimla with chief complaint of missing canine on left side and retained deciduous canine on right side of the maxillary arch. A review of her medical and dental histories revealed no significant findings or contraindication to dental care. The extraoral clinical examination disclosed balanced vertical third and an orthoganathic profile. The intraoral examination revealed class I intercuspation on both side with retained deciduous right canine and missing canine with sufficient space on left side (Figure 1A-1D). Canine bulges were visible and palpable on right side palatally and on left side labially just above the left lateral incisor. The radiographic examination disclosed palatally impacted right maxillary canine and labially impacted canine on left side with almost complete resorption of the root of left lateral incisor (Figure 2A-2B).

Since the patient was dento-skeletally class I with well aligned lower arch with perfect class I posterior occlusal intercuspation and normal overjet-overbite, so the treatment was planned to treat the upper arch only by moving the impacted canine orthodontically into the arch.

Treatment was explained to the parents regarding surgical exposure of the impacted canines and the poor prognosis of left leteral incisor

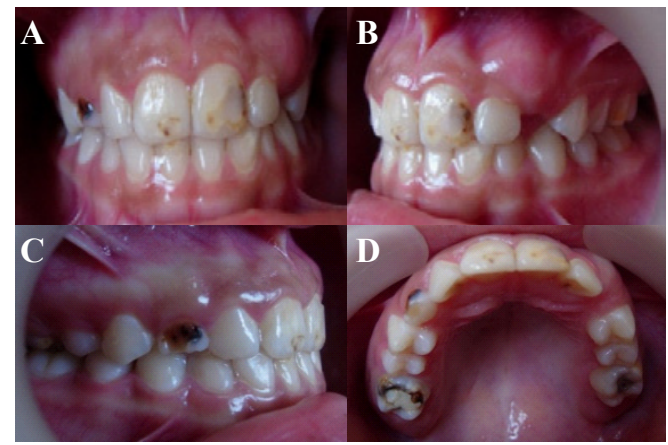

Figure 1: Pre-treatment intra-oral photographs showing class I occlusion with retained right deciduous canine and missing left canine. 
Citation: Negi KS (2012) Bilateral Impacted Maxillary Canine with Left Lateral Incisor Root Resorption. Dentistry 2:134. doi:10.4172/21611122.1000134

due to almost complete resorption of its root by the labially impacted canine.

Upper arch bonded with 022 pre-adjusted edgewise appliance except the left upper lateral incisor. After the alignment and leveling, arch was prepared for surgery. A full thickness flap elevation was performed exposing only enough crowns to allow careful debridement and the lingual buttons were bonded to the exposed crowns (Figure 3A-3D). The retained deciduous right canine was extracted. The ligature wire attached to lingual button were pierced through the flap and ligated to the archwire then the flap was repositioned and secured with interrupted 4-0 gut suture. After five months of orthodontic treatment the left canine aligned in the arch and right canine shows remarkable movement and change its position in orthopantomogram (Figure 4A-4C). On eighth month right canine was visible through the palatal mucosa and now the extrusive force was applied by archwire with stepdown bend with piece of elastomeric chain (Figure 5). After a month complete canine was visible, ligual button removed and the bracket bonded in proper position on the labial surface of the canine and 016" NiTi alignment archwire was ligated. After the alignment $016 \times 022 \mathrm{NiTi}$ archwire was ligated for levelling and then 019x022 Stainless steel archwire was ligated and left for four month for proper root positioning. Case was debonded after the radiographic confirmation of proper root position and a canine to canine bonded retainer was given (Figure 6A-6E). In the end of treatment a well formed arch with both impacted canines in proper position and normal class I intercuspation was achieved except the left lateral incisor which was in compromised position due to root resorption. The compromised left lateral incisor which is splinted with canine to canine lingual bonded retainer is fulfilling the esthetic requirement long as it can and

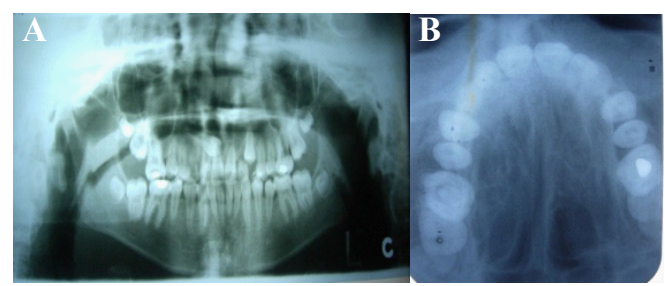

Figure 2: OPG and Occlusal radiograph showing palatal impaction of right canine and labial impaction of left canine with root resorption of lateral incisor.
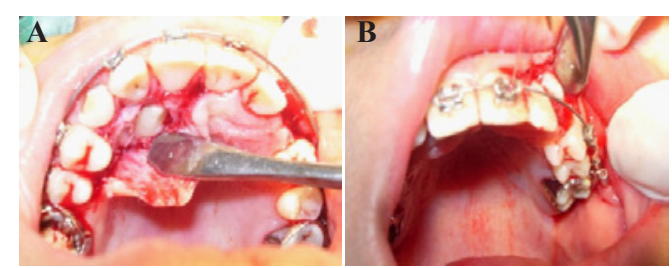

C

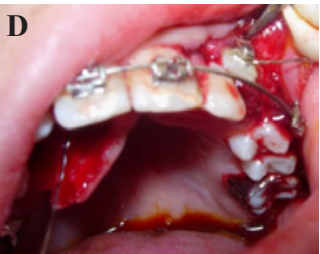

Figure 3: Intra-oral photograph showing surgical exposure of impacted canines.

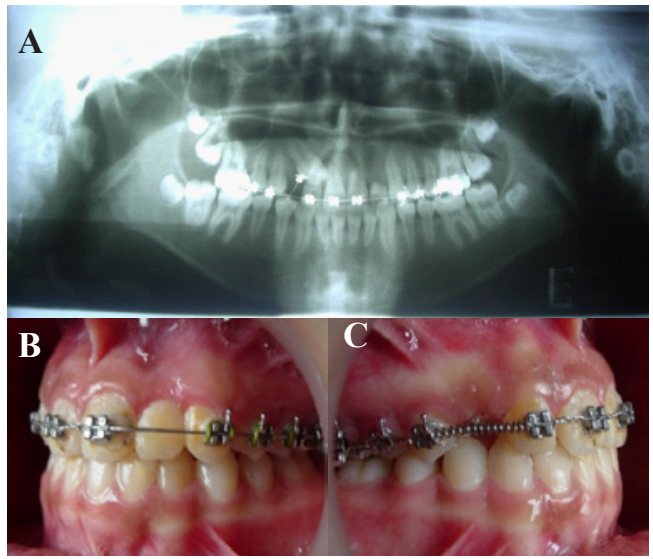

Figure 4: Intra-oral photograph and OPG showing the position of canine after 5 months of orthodontic treatment.

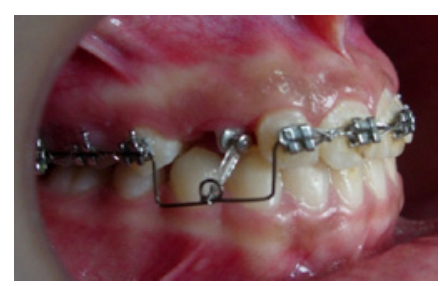

Figure 5: Intra-oral photograph showing the orthodontic eruption of right palatally impacted right canine after 8 months of treatment.
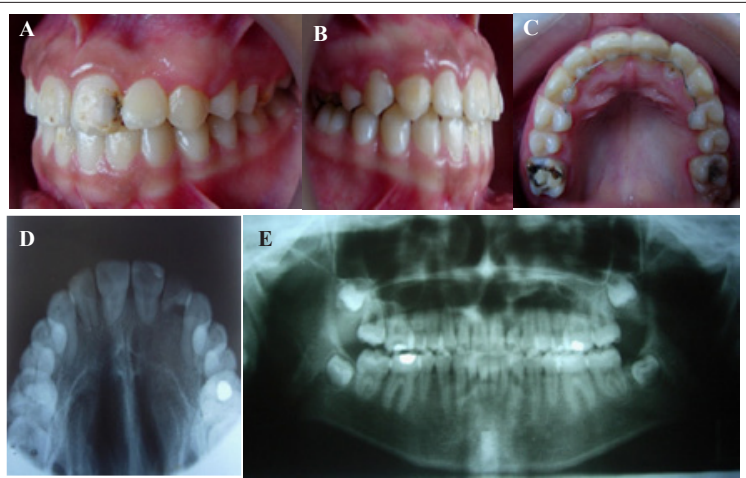

Figure 6: Post- treatment intra-oral photographs and radiographs showing well aligned and positioned impacted right and left canines.

patient was conveyed for the prosthetic replacement later on. Patient was advised to go for re-restoration of 11,16 and 12 which were not properly restored.

\section{Discussion}

The management of impacted canines is important in terms of esthetics and function. Clinicians must formulate treatment plans that are in the best interest of the patient, and they must be knowledgeable about the variety of treatment options. When patients are evaluated and treated properly, clinicians can reduce the frequency of ectopic eruption and subsequent impaction of the maxillary canine. The simplest interceptive procedure that can be used to prevent impaction of permanent canines is the timely extraction of the primary canines. This procedure usually allows the permanent canines to become 
Citation: Negi KS (2012) Bilateral Impacted Maxillary Canine with Left Lateral Incisor Root Resorption. Dentistry 2:134. doi:10.4172/21611122.1000134

upright and erupt properly into the dental arch, provided sufficient space is available to accommodate them, which is not been followed in this case, must be the main etiological factor of impaction and root resorption due ectopically placed left canine. Various surgical and orthodontic techniques may be used to recover impacted maxillary canines. The proper management of these teeth, however, requires that the appropriate surgical technique be used and that the clinician be able to apply measured forces in a favorable direction. This allows for complete control in efficient correction the impaction and for avoidance of damage to adjacent teeth. Careful selection of surgical and orthodontic techniques is essential for the successful alignment of impacted maxillary canines.

Treatment of case that presented with impacted maxillary canine and extensive resorption of root of left lateral incisor is reported. Treatment consisted of non extraction protocol with surgical exposure and orthodontic repositioning of canines. A recent report highlighted the advantage of closed eruption technique when compared with the apically repositioned flap technique. For this patient, however, none of the negative sequelae associated with apically positioned flap (poor esthetics, increased crown length, and intrusive relapse) were clinically significant.

\section{Conclusion}

Pediatric dentists and general practitioners should familiarize themselves with this dental anomaly and be trained to detect teeth at risk for impaction. Early diagnosis and subsequent precautionary measures, such as primary canine extraction, may prevent further complications and spare patient time, expense and complex treatment procedures. A proper diagnosis and careful selection of surgical and orthodontic technique help to achieve a successful alignment of impacted canines.

\section{References}

1. Power SM, Short MB (1993) An investigation into the response of palatally displaced canines to the removal of deciduous canines and an assessment of factors contributing to a favourable eruption. Br J Orthod 20: 215-223.

2. Bedoya MM, Park JH (2009) A review of the diagnosis and managementof impacted maxillary canines. J Am Dent Assoc 140: 1485-1493.
3. Ngan P, Hornbrook R, Weaver B (2005) Early timely management of ectopically erupting maxillary canines. Semin Orthod 11:152-163.

4. Bishara SE (1992) Impacted maxillary canines: a review. Am J Orthod Dentofacial Orthop 101: 159-171.

5. Cooke J, Wang HL (2006) Canine impactions: incidence and management. Int J Periodontics Restorative Dent 26: 483-491.

6. Proffit WR, Fields HW, Sarver DM (2007) Contemporary Orthodontics (4thedn), St. Louis: Mosby.

7. Yavuz MS, Aras MH, Buyukkurt MC, Tozoglu S (2007) Impacted mandibular canines. J Contemp Dent Pract 8: 78-85.

8. Ericson S, Kurol J (1988) Early treatment of palatally erupting maxillary canines by extraction of the primary canines. Eur J Orthod 10: 283-295.

9. Mitchell L (2007) An Introduction to Orthodontics. (3rdedn), New York.

10. Jacoby $H$ (1983) The etiology of maxillary canine impactions. Am J Orthod 84 125-132.

11. Becker A (2007) The Orthodontic Treatment of Impacted Teeth. (2ndedn) Abingdon, Oxon, England.

12. Peck S, Peck L, Kataja M (1994) The palatally displaced canine as a denta anomaly of genetic origin. Angle Orthod 64: 249-256.

13. Baccetti T (1998) A controlled study of associated dental anomalies. Angle Orthod 68: 267-274.

14. Ericson S, Kurol J (1988) Resorption of maxillary lateral incisors caused by ectopic eruption of the canines: a clinical and radiographic analysis of predisposing factors. Am J Orthod Dentofacial Orthop 94: 503-513.

15. Rimes RJ, Mitchell CN, Willmot DR (1997) Maxillary incisor root resorption in relation to the ectopic canine: a review of 26 patients. Eur J Orthod 19: 79-84.

16. Liu DG, Zhang WL, Zhang ZY, Wu YT, Ma XC (2008) Localization of impacted maxillary canines and observation of adjacent incisor resorption with conebeam computed tomography. Oral Surg Oral Med Oral Pathol Oral Radiol Endod 105: 91-98.

17. Maverna R, Gracco A (2007) Different diagnostic tools for the localization of impacted maxillary canines: clinical considerations. Prog Orthod 8: 28-44.

18. Walker L, Enciso R, Mah J (2005) Three-dimensional localization ofmaxillary canines with cone-beam computed tomography. Am J Orthod Dentofacial Orthop 128: 418-423.

19. Elefteriadis JN, Athanasiou AE (1996) Evaluation of impacted canines by means of computerized tomography. Int J Adult Orthodon Orthognath Surg 11 257-264. 